\title{
Application Research of Integrated Experimental Platform in Industrial Design Model Making
}

\author{
Yang Jiaxin', Yang Guang ${ }^{2}$ \\ ${ }^{1}$ Department of product design, Harbin University of Commerce, Harbin 150028, China \\ ${ }^{2}$ Department of environmental art design , Harbin University of Commerce, Harbin 150028, China
}

Keywords: Industrial design; model making; integration; experimental platform;

\begin{abstract}
In order to improve the overall level of the experimental teaching in the industrial design model of colleges and universities, provide more effective knowledge reserve to the process of industrial design, and formulate an organization system based on the integrated experimental platform, so that the design of industrial products can be stimulated by leaps and bounds. To coordinate the practical teaching technology mechanism, implement the integrated project based on the reorganization and advance, change the relationship between the model production and practice teaching, set up the reasonable teaching content and method, pay attention to the combination of theory and practice, carry out the comprehensive attempt of the reform of the mode, study and cultivate the experimental teaching model of the industrial design course group. Quality compound talents have laid an important material foundation for designing, evaluating and innovating.
\end{abstract}

\section{工业设计模型制作中集成化实验平台的应用研究}

\author{
杨佳欣 ${ }^{1}$ ，杨光 ${ }^{2}$ \\ 1.哈尔滨商业大学产品设计系, 黑龙江 哈尔滨 150028 \\ 2. 哈尔滨商业大学环境艺术设计系，黑龙江 哈尔滨 150028
}

关键词:工业设计; 模型制作; 集成化; 实验平台;

摘 要: 为了完善高校工业设计模型制作实验教学的整体水平，给工业设计过程提供更有效的 知识储备, 制定出一种基于集成化实验平台的组织体系, 从而使工业产品设计得到跨越式激 励。协调实践教学技术机制, 实施基于重组并进的集成项目, 转变模型制作实践教学的相互 关系, 设置合理的教学内容和方法, 注重理论与实践相结合, 开展方式改革的综合尝试, 为 工业设计课程群实验教学模式的研究以及培养高素质复合型人才从事设计、评价、创新工作 奠定了重要的物质基础。

\section{1. 引 $\square$ 言}

信息技术时代对现代设计人才需求的批量化,逼迫着工业设计产业教育, 要尽快为社会提供 必须的现代化人才，追其根源是适当的创新实验人才培养模式没有形成、实践教育课程与实 验平台尚未融合、创新实验平台的应用尚未形成明确目标这三方面。

工业设计实践教学的具体表达，可以通过建立集成化过程来表现高水平的创意产品。模型 制作作为重要必修课程, 贯穿于整个专业学习始终, 并需要完全依赖于模型制作实验室, 因 此实验平台建设的质量高低直接影响到产品模型制作的好坏。目前很多高校在模型制作实验 室平台建设上相对混乱，无法满足教学要求，本文将此为重点进行探究,并提出自己的见解。 


\section{2. 模型制作实验平台的基础建设}

\section{1 模型制作实践教学的现状}

就传统工业设计教学而言, 实验教学课程质量和最终成果往往和理想目标存在很大差距, 针对实验教学集成化平台体系的建构组合, 必会是完善工业设计复合型人才培养的重要媒介。 从目前本科教学的模型制作课程中涵盖实验教学课程内容的实际案例进行纵向的集成化分 析, 可以看出其极大的应用实践比例。虽然在教学改革整体发展中, 设计理论类教学发展在 这几年得到了长足进步和完善, 但是对于实验教学, 仍被单纯作为设备采购和单一对应课程 的初始阶段。

通过架构设计理论和设计实践相结合的科学教学体系来推行集成化实验教学, 明确在当前 创意产业背景下实验教学集成化的概念和属性, 总结出高校工业设计实验教学中的阶段性启 示, 对于设计人才挖掘最新设计理念和技术有着更深层次的支撑力。提出推广和深化实验教 学的思路, 既可以被认为是课程教学体系中的纵向实验, 也可以被应用于实验平台的横向构 建, 最终达到切实提高设计专业教学质量的目的。

\section{2 模型制作实验平台的建设路径}

\subsection{1 按不同材料与加工工艺的需要建设}

产品模型制作能选用的材料很多, 主要选用易于加工, 具有一定强度, 不易变形的材料。 按照选用的材料制作模型可分为, 油泥模型、粘土模型、木材模型、卡纸模型、塑料模型、 石膏模型、玻璃钢模型、金属模型等。因此需要按照不同模型材料加工的需要, 建设不同类 型的实验制作室，例如陶泥模型制作室、石膏模型制作室、木工模型制作室、塑料模型制作 室、玻璃钢模型翻制室、金属模型制作室等, 将其整合起来, 通过对材料的综合加工利用, 组成具有实践价值的工业模型制作实验室。

\subsection{2 按不同设计原则与用途的需要建设}

设计的复杂性和多样性对模型制作提出了更高的要求, 时代的变化和科技的进度使模型的 原则内涵更加丰富, 外延的能力也变得更加宽广。根据设计原则用途的不同, 模型包括参考模 型、结构功能模型、展示样品模型。参考模型同属于初级模型, 由构思模型和概念模型组成; 结构功能模型同属于中级模型, 由结构模型和功能模型组成; 展示样品模型同属于高级模型, 由外观模型、产品展机和产品样机组成。三者综合对应重构, 组成工业设计模型制作实验室。

\section{3. 模型制作中实验教学集成化平台的要素构建}

集成化平台构建是为了更好地开展实验教学的规范化研究。在明确了工业设计实验教学的 重要意义之后, 通过有技术性、科学性、针对性的三维实验教学集成化模块体系的形成, 架 构出更为立体有效的出发点群落则是整体教学的重中之重。基于上述引入集成化平台的核心 理念, 探讨出三点集成样本的构成要素。

首先是设计感知教学要素, 该要素着眼点是设计讨论教学的感知阶段, 将设计形象转化为 产品形象时, 纵观工业设计各课程体系的整体排布和标准化目标, 设计感知教学本应被称为 课程集成化平台的基石。该学时阶段不仅是单一的SET因素分析法, 亦或是头脑风暴等基础 设计思维方法的集合, 是要从主观理念突破分析视角, 以更真实地实验样本数据着手, 形成 金字塔集成化的转化框架。通过金字塔板块要素的自助式服务, 进行顶端端点更正确的把握。

其次是实验选题及反推教学要素, 在初级集成化选择之后, 第二阶段是对核心理念更深层 次的发掘操作。比如人机工程学、各年龄段心理测试、医疗辅助设备采集等方面, 运用工业 设计实验室各个设备进行实践, 体现其灵活适应性, 并最终运用大数据解析样本, 完备该群 体的设计定位, 以达到实验选题集成化教学平台的准确度再生。 
实验选题教学过程中所出现的前期成果, 往往会由于未知某些因素影响到产品效果, 大多 数情况下, 会被隐藏的细节性设计所掩盖, 这也正是惯性思维教学手法的弊端。此时便需要 反推教学要素, 将产品后期所反馈的测试结果进行逆推, 设计中心基准点的校对是要点。制 作模型可以利用行为测定仪、眼动仪等实验设备, 来检测产品基本色彩、形态、肌理、结构 等专业数据的集成化采集。由此可形成相关实验样品数据库, 可表现的问题进行反证收集, 形成样本平台。

最后则是重组并进教学要素, 在每节设计模型制作课程结束所进行的课程总结中, 按照不 同设备的选用、使用率、使用时长等现实因素, 系统地进行有效重组排列, 从而使工业设计 实验室效率集成化平台能正确地把握市场动向, 通过整体课程周期性样本库研究, 对新理论 体系中涵盖的实验技术进行精密升级和淘汰, 或从高段教学设计到普及基础教学设计的普遍 性实验展开。力求该平台在整个工业设计实验教学课程中的前端性、有效性、融合性得到最 大程度的深化。

\section{4. 基于集成化实验平台的模型制作教学实践}

传统模型制作手段是根据设计成型的生产方式为依托, 而集成化模型制作实验平台是以智 能化的生产方式为依托。由此两种生产方式的实际改变, 将模型制作实验平台从技术化的角 度进行了革新。建立多维化的模型制作实验技术工艺流程, 增加3D打印集成化模型制作平台, 实现从单一实验模式向多元化实验模式的转变。学生通过新搭建的集成化多系列技术实验平 台, 找寻到适合制作对象的造型、功能等因素, 选择某种实验技术, 根据该技术完成图纸表 现、加工工艺、质量检验等实验内容。

模型制作集成化平台关键部分就是制定结构、教学顺序以及规划。根据课程设置整合后的 模型制作技术平台，两个综合系列课程，六个单项实践结构。第一是以3D打印技术为集成化 实践课程，第二则是以模具成型技术为集成化实践课程。两者均需要从计算机基本视图设计、 建模数据分析、3D立体打印成型、表面涂饰和细节表现、零部件装配等各单项内容去划分, 学生可以自主选择其一，也可按照产品造型与成型工艺的难易程度去交叉运用。

\section{5. 结 论}

工业设计模型制作集成化实验平台设计需从两个并行的、具有内在联系的步骤开始, 即课 程结构的设置和教学顺序的确定, 再把教学顺序标注到课程结构的各要素之中; 首先这样的 集成化课程平台相互支撑、互相联系, 每个要素都在实践教学中起到明确作用; 其次从集成 构造要素角度, 集成物质系统手段, 集成实践价值三方面, 列为实践教学研究内容进行系统 深入的梳理, 从而推行集成化创新创业课程体系设计关键; 再次, 集成化实验平台系统的建 设只是提供了一个硬件平台, 更关键的问题是 “软件”, 即实验内容的建设,这方面的探索还需要 更深入的探索。最后, 该实验平台对学生自觉性要求较高, 学生需自主讨论、研究, 找寻出 解决问题的途径和手段, 体现了学生学习的主体性, 学到知识的同时, 也收获了实际项目制 作的经验。

\section{[参 考 文 献]}

[1] 庄孔韶. 人类学概论 [M]. 北京: 中国人民大学出版社, 2006.

[2] 陈昌曙. 技术哲学引论[M]. 北京: 北京科学出版社, 2012.

[3] 滕守尧等. 后工业化会的设计、文化与技术[M]. 成都：四川人民出版社，1998.

[4] [德] 乌尔里希. 森得勒. 工业 4.0 即将来袭的第四次工业革命[M]. 北京: 机械工业出版 译, 2014. 
[5] [美] 杰里米·里夫金. 第三次工业革命一新经济模式如何改变世界[M]. 北京：中信出版 社译, 2012.

[6] [美] 丹尼尔 $\cdot F \cdot$ 库法罗等. 工业设计技术标准常备手册 $[M]$. 上海：上海人民出版社译， 2009.

[7] 刘新. 关于设计评价标准的思考[C]. 北京：中国科学技术协会编, 2007.

[8] [英] 约翰. 克里斯托弗·琼斯. 一种系统设计的方法[C]. 北京: 凤凰传媒集团出版译, 2010.

[9]［英］彼得·戈伯. 设计的未来及其管理[C]. 江苏：江苏美术出版社译，2010.

[10] 王敏. 产品造型设计的 A E T 三维评价研巧[D]. 杨凌：武汉理工大学, 2013.

[11] 刘兴波. 关于高校教学过程中技能问题的研究[J]. 教育探索，2015，(5)：32-38.

[12] 周俊等. AXDE 教学模式在经管类专业课程实践教学中的应用 $[\mathrm{J}]$ 职教论坛, 2015, 6(3): $10-18$.

[13] 薛玉香, 王占仁等. 地方高校应用型人才培养特色研究[J]. 高等工程教育研究, 2016(1): $149-153$.

[14] 崔玉平. 高校创新创业教育改革的经济意义和行动条件[J]. 南京师大学报(社会科学版), 2016(5): 85-93.

[15] 李保军, 唐晓红等. 高等院校化学专业青年教师提高教学能力的措施[J]. 河南教育学院 学报(哲学社会科学版), 2017(5): 20-31.

[16] 陈建勇, 徐定华, 关勤. 省级实验示范中心在高校人才培养中的作用 $[\mathrm{J}]$. 科技资讯, 2014(10): 57-60.

[17] Zhao, C., Z. Liu. Developing Interdisciplinary Design Curriculum Cube Model: A Constructivism Perspective [A]. in 1 st International Conference of Design Creation and Practice[C]. 2010. Taibei:Sun Good Publisher.

[18] Papanek, V. Design for the Real World : Human Ecology and Social Change [M]. 2nd ed. London: Thames and Hudson, 1985.

[19] Lawson, B. How Designers Think. The Design Process Demystified[M]. Fourth edition ed. Burlington: Architectural Press, 2015.

[20] Cross, N. Designerly Ways of Knowing[M].Springer, 2016. 